\title{
Conventional and variant termination of the portal vein in a black Kenyan population
}

\author{
J. Munguti, K. Awori, P. Odula, J. Ogeng'o \\ Department of Human Anatomy, University of Nairobi, Kenya \\ [Received 7 July 2012; Accepted 11 August 2012]
}

Background: Knowledge of the variant terminations of the portal vein (PV) is important in surgical and interventional radiological procedures of the liver. Their pattern and frequency are important in planning surgery to minimise complications. They differ between populations, but data from Africa is unavailable. The aim of the study is investigate the variant termination of the main PV.

Materials and methods: One hundred livers from adult black Kenyans (age range 35-79 years) were studied at the Department of Human Anatomy, University of Nairobi, Kenya by gross dissection. The livers were cleared of blood, fixed with 10\% formaldehyde solution, and the venous system infused with acrylate monomers to keep the veins firm. The level of termination relative to the capsule and the branching pattern of the PV were examined. Various patterns were photographed using a digital camera. Data was analysed using SPSS version 16.0 for windows and presented in tables and macrographs.

Results: Portal vein termination was extracapsular in 14\%, capsular in 40\%, and intrahepatic in $46 \%$ of examined cases. Variant termination occurred in $49 \%$ of cases while conventional bifurcation occurred in $51 \%$ of cases. The level of termination of the PV had a positive correlation with its pattern $(p=0.05)$.

Conclusions: The high prevalence of variant termination of PV in the current study suggests that the population is more vulnerable to inadvertent injury during surgery and radiological intervention. Moreover, an extra hepatic termination of the PV is more likely to have a conventional branching pattern compared to an intra-hepatic termination. (Folia Morphol 2013; 72, 1: 57-62)

Key words: bifurcation, transjugular, variations, hepatectomy

\section{INTRODUCTION}

The portal vein (PV) usually terminates by bifurcating into the right and left PV branches. Other termination patterns include a bifurcation into a common left PV trunk and the right anterior PV trunk; trifurcation; and quadrifurcation $[2,11]$. These terminations may be extracapsular, capsular, or intrahepatic [17]. Variations in level and pattern of termination influence the risk of vascular injury dur- ing procedures such as hepatectomies, split or living donor transplantation, PV embolisation, and the placement of transjugular intra-hepatic portosystemic shunts (TIPS) [13]. Knowledge of these variations decreases complication rates in these procedures and is also important in identifying the location of liver lesions $[6,7]$. These variations also increase the risk of bile duct hilar anatomical variation [16]. Furthermore, the branching pattern de-

Address for correspondence: J. Munguti, Department of Human Anatomy, University of Nairobi, P.O. Box 30197 00100, Kenya, tel: +254-727161449, e-mail: Donaldjrmh86@gmail.com 
termines the orientation of the branches, which is vital for surface mapping of the liver during hepatic segmentectomy [1]. The frequency of these variations displays population differences [12, 14, 17]. Data from African populations is, however, unavailable. This study therefore examined the level and pattern of termination of the main trunk of the portal vein (MPV) in a black Kenyan population.

\section{MATERIALS AND METHODS}

One hundred livers, obtained between 2010 and 2011 from adult black Kenyans during autopsy and dissections at the Department of Human Anatomy, University of Nairobi, Kenya were used in this study. Ethical approval for the study was granted by Kenyatta National Hospital-University of Nairobi Ethics and Research Committee. Cirrhotic livers and those harvested from individuals with abdominal injuries involving the liver and the portal system or history of previous hepatic and pancreatic surgery were excluded from the study. Livers which the pathologist had cut during autopsy were also excluded.

Once the abdomen was opened and its viscera exposed, the hepatogastric and hepatoduodenal ligaments were cut to separate the liver from the stomach and the duodenum. The stomach was then retracted laterally. The duodenum and the coils of small intestines were retracted posteroinferiorly to expose the head of the pancreas, which was then carefully retracted laterally to expose the location of the MPV formation. Once identified, the PV was followed superiorly along the right free border of the lesser omentum up to the porta hepatis. The liver was subsequently detached from the anterior abdominal wall by incising the falciform ligament, and from the diaphragm by incising the triangular and the falciform ligaments. The PV was cut close to its formation and the whole liver lifted from the abdominal cavity with the vein attached.

The liver was cleared of clotted blood and fixed by infusing $10 \%$ formaldehyde solution through the PV for 10 min. Acrylate monomers (from Dawa pharmaceuticals, Kenya) were mixed and infused into the PV. The liver was kept for 3 days at $4^{\circ} \mathrm{C}$ for the monomers to set while keeping the liver soft.

To determine the level of termination of the PV, the common hepatic duct, common hepatic artery, and the connective tissue surrounding them were dissected away and the PV followed to the porta hepatis. Termination was taken to be extracapsular if there was an interval between the point of PV termination and the liver surface. Capsular termination, on the other hand, was one in which the PV termination was adherent to the liver surface. If part of the liver had to be dissected to reveal the PV termination, this was defined as intra-hepatic termination.

The branching pattern of the PV was classified after Atasoy and Ozvurek [2], as follows: Type 1 conventional bifurcation; Type 2 - variant bifurcation in which the PV gave the right anterior PV and a common trunk from which arose the left PV and right posterior PV; Type 3 - trifurcation if it gave three branches - the right anterior, right posterior and the left portal veins. In addition, we included what we called type 4, that is: quadrifurcation if it gave, besides the branches observed in Type 3, a branch to the caudate lobe whose diameter was at least a third of that of the PV. All observations were recorded and photographs taken with a highresolution digital camera. Observations to determine the branching pattern were each made by two different persons to counter intra-observer variability. The results are presented in a table, a bar graph, and macrographs.

The pattern and level of termination of the PV were compared to establish whether or not there was a correlation using Pearson's Correlation - Free Statistics Software (Calculator) [18].

\section{RESULTS}

A single PV was present in all the cases and entered the liver at the porta hepatis together with common bile duct and hepatic artery proper. It lay posterior to both structures. However, it displayed variations in level of termination and terminal branching pattern.

\section{Level of termination of the portal vein}

The termination of the PV was extrahepatic in $54 \%$ of the cases. Of these, $40 \%$ were capsular termination while the rest (14\%) were extracapsular termination. Intrahepatic termination occurred in $46 \%$ of the total study population (Fig. 1). The conventional termination of the PV (Type 1) occurred in $51 \%$ of cases while Type 2 pattern of termination had a prevalence of $15 \%$. The prevalence of Types 3 and 4 was $22 \%$ and $12 \%$, respectively (Fig. 2). When the pattern and level of termination of the PV were compared in the current study, the correlation coefficient was 0.95 $(p=0.05)$. The comparisons are shown graphically in Figure 3. 

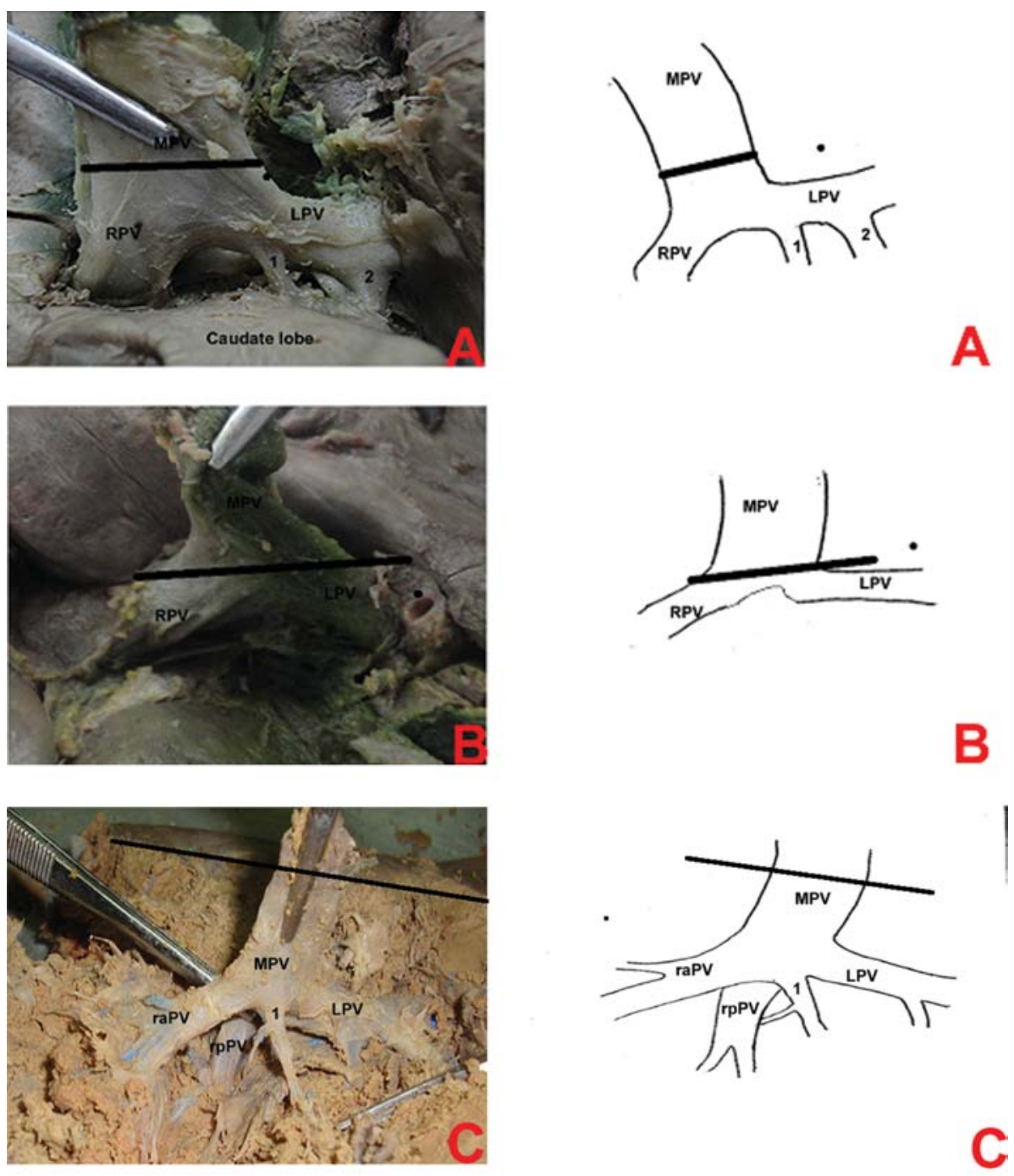

Figure 1. Level of termination of PV; A. An extracapsular Type 1 PV termination. The bold line indicates the position of the capsule before it was dissected out. Note the distance of the level of bifurcation from the hepatic surface. Branches to the caudate lobe are labelled 1 and 2; B. Capsular type 1 PV termination. The bold line indicates the position of the capsule before it was dissected out; C. Intrahepatic Type 4 PV termination. Part of the liver has been dissected out to reveal the point of termination. The bold line indicates the location of the hepatic surface before dissection; PV — portal vein; MPV — main trunk of the portal vein; RPV — right portal vein; LPV — left portal vein; raPV — right anterior portal vein; rpPV — right posterior portal vein; 1 and 2 — branches to caudate lobe.

\section{DISCUSSION}

The portal venous trunk shows variations in its level and pattern of branching [1]. Such variations increase the risk of vascular injury during surgical procedures such as hepatectomies; split or living donor transplantation and other complex interventional procedures such as PV embolisation and the placement of TIPS [13].

Observations of the present study have revealed that PV is present in all cases. Indeed, as reported, its absence is rare [10]. Similar to accounts in the literature, variations occur frequently in the level and pattern of termination of the PV [1].
The level of termination of the PV determines the position of shunt placement during TIPS. In the current study, $54 \%$ of the cases terminated extra hepatic $(n=100)$, higher than $47 \%(n=70)$ reported for a Chinese population [12], and $48.4 \%(n=57)$ for a Caucasian population [17]. In $46 \%$ of the cases the termination was intrahepatic. This is higher than the figure of $26 \%$ reported for the Caucasian population [17]. Capsular termination occurred in $40 \%$, higher than the figure of $25.8 \%$ reported for the Caucasian series [17]. From these studies, which were all cadaveric, there appear to be wide variations in the level of PV termination. Although the sample size may explain 

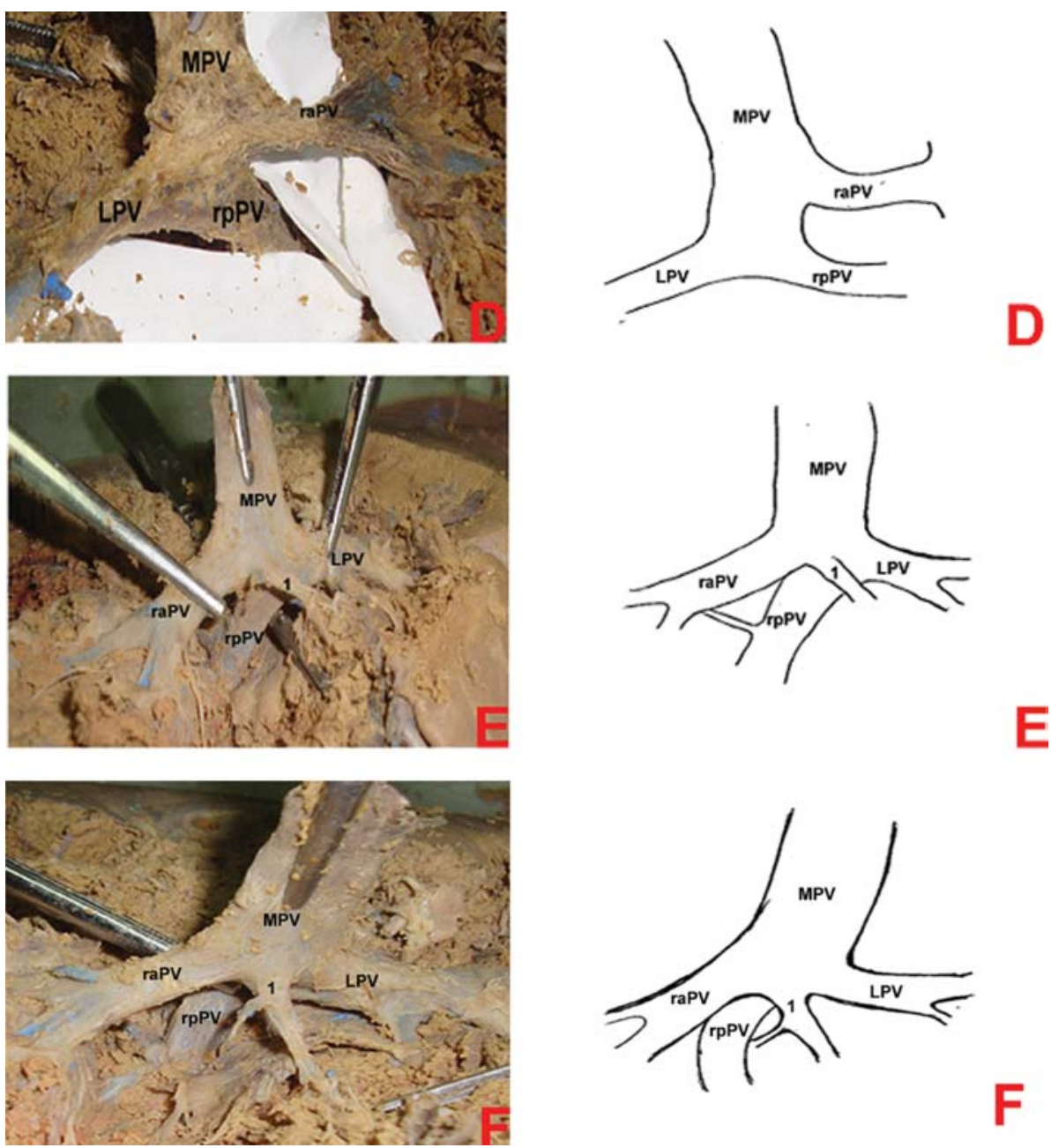

Figure 2. Pattern of termination of PV; D. Intrahepatic Type 2 PV termination. Note the common LPV trunk which gives rise to the LPV and $\mathrm{rpPV}$; E. Intrahepatic Type $2 \mathrm{PV}$ termination. The rpPV was given off the trunk, which continued as the LPV; F. Intrahepatic Type 4 PV termination. The PV gave a big branch (1), which was the only source of blood to the caudate lobe; PV — portal vein; MPV — main trunk of the portal vein; RPV — right portal vein; LPV — left portal vein; raPV — right anterior portal vein; rpPV — right posterior portal vein; 1 and 2 — branches to caudate lobe.

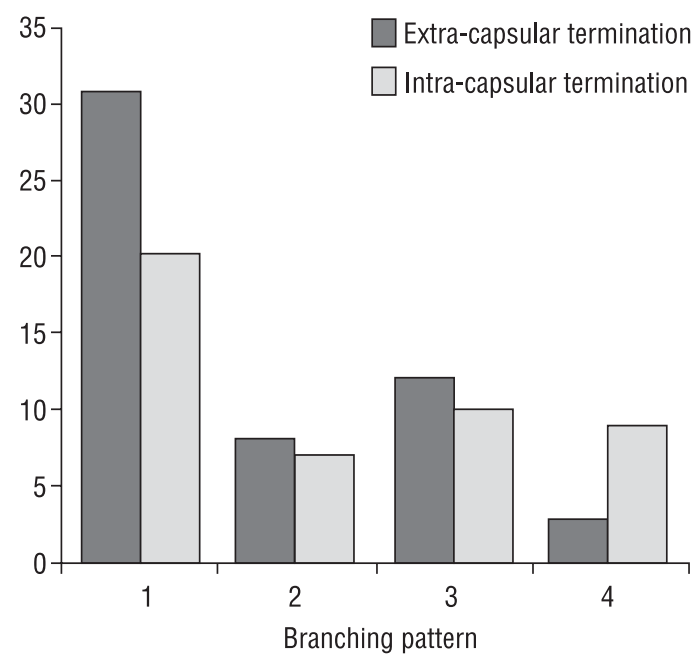

Figure 3. Comparisons between pattern and level of termination of the portal vein. the disparity, it is probable that actual ethnic differences exist.

A correlation coefficient of $0.95(p=0.05)$ for the level and pattern of termination shows that there is a strong positive influence of the level of termination of the PV on its pattern of termination. Thus, an extra-capsular termination is more likely to give a conventional pattern of termination compared to an intra-capsular one. Similarly, a variant PV termination is more likely to occur in an intra-capsular termination than in an extra-capsular termination. This should be borne in mind when performing liver surgical procedures.

Anatomical variations in the branching of the PV are relatively common $[2,6]$. The most remarkable observation of the current study is the low preva- 
Table 1. Comparison of pattern of termination of main trunk of the portal vein in different populations

\begin{tabular}{lccccccc}
\hline Author, year & Population & Methodology & Sample size & \multicolumn{4}{c}{ Frequencies of the branching pattern (\%) } \\
\cline { 5 - 7 } & & & & Type 1 & Type 2 & Type 3 & Type 4 \\
\hline Gupta et al., 1977 [9] & Indian & Cadaveric & 85 & 88 & - & 12 & - \\
Park et al., 1996 [15] & Korean & CT angiography & 632 & 73.9 & 8.4 & 16.1 & 0.8 \\
Koc et al., 2007 [11] & Turkish & MDCT & 1384 & 72.6 & 9.7 & 11.1 & 0.2 \\
Covey et al., 2004 [6] & American & CT angiography & 200 & 65 & 9.0 & 13 & 7 \\
Carr et al., 2003 [4] & American & MR angiography & & 76 & 16 & 8 & - \\
Current study & Kenyan & Cadaveric & 100 & 51 & 15 & 22 & 12 \\
\hline
\end{tabular}

CT — computed tomography; MDCT — multi-slice computed tomography; MR — magnetic resonance

lence $(51 \%)$ of Type 1 mode of termination compared to prevailing reports in literature (Table 1). Type 1 termination is associated with reduced risk during hepatic procedures [16]. This suggests that the Kenyan population is more vulnerable to injury during surgery and radiologic intervention. The $49 \%$ variant termination in the present study is significantly higher than the $10-35 \%$ sonographic and computed tomography portographic reports found in literature $[3,4,6,8]$ for Oriental and Caucasian populations (Table 1). Although the current study is based on gross dissections, which might explain this difference, the previous studies used imaging techniques reported varying incidences. It therefore implies that methodological differences alone cannot explain this disparity, raising the possibility of an ethnic influence.

Type 2 termination occurred in $15 \%$ of the cases, higher than the reported figure of $8.4 \%$ from Korean and $9.7 \%$ from Turkish populations. It is nonetheless lower than the $23.5 \%$ reported in the Turkish population [11]. These results reveal wide variations in the prevalence of Type 2 , again suggesting possible ethnic variations. However, we report a lower incidence of Type 2 branching pattern compared to the other variants since it has previously been reported as the most frequent [16].

Both Types 3 and 4 are much more prevalent than those previously reported in literature (Table 1). The modes of termination are important in PV embolisation during percutaneous interventional procedures, liver transplants, segmentectomy, and PV embolisation [6]. This implies that nearly $50 \%$ of the black Kenyan population may be unsuitable liver donors [5] and are more vulnerable to hepatic injury and perforation during such procedures [7].

\section{CONCLUSIONS}

Variant termination of PV occurs in $49 \%$ of the study population, much higher than prevailing reports. This suggests that the population is more vulnerable to inadvertent injury during surgery and radiological intervention. Extra diligence should be exercised during procedures on the liver. Preoperative portal venography evaluation is thus emphasised. These observations also indicate that an extra-hepatic termination of the PV is more likely to have a conventional branching pattern compared to an intra-hepatic termination.

\section{RECOMMENDATIONS}

We recommend that future studies involving the level and pattern of MPV termination in a black Kenyan population be done using imaging studies. This would enable a closer comparison of these results with those from other populations, conducted in the recent past, employing imaging methods.

\section{REFERENCES}

1. Arora J, Kapur V, Kakkar A, Dixit PC (2003) Ramification of portal vein in right lobe of liver: a corrosion casting study. J Anat Soc India, 1: 12-14.

2. Atasoy C, Ozvurek H (2006) Prevalence and types of main and right portal vein branching variations on MDCT. AJR, 188: 492-497.

3. Atric M, Bret PM, Fraser-Hill MA (1992) Intrahepatic portal venous variations: Prevalence with ultrasound. Radiology, 184: 523-526.

4. Carr JC, Nemcek AA Jr, Abecassis M, Blei A, Pereles FS, Mc Carthy R, Finn JP (2003) Preoperative evaluation of the entire hepatic vasculature in living liver donors with use of contrast enhanced MR angiography and time fast imaging with steady: state precession. J Vasc Interv Radiol, 14: 441-449.

5. Cheng YF, Huang TL, Lee TY, Chen TY, Chen CL (1996) Variation of intrahepatic portal vein; angiographic 
demonstaration and application in living related hepatic transplantation. Transplant Proc, 28: 1667-1668.

6. Covey AM, Brody LA, Getrajdman GI, Sofocleous CT, Brown KT (2004) Incidence, patterns, and clinical relevance of variant portal vein anatomy. AJR, 183: 1055-1064.

7. Erbay N, Raptopoulos V, Pomfret EA, Kamel IR, Krinskal JB (2003) Living donor liver transplantation in adults: vascular variants important in surgical planning for donors and receipients. AJR, 181: 109-114.

8. Filly RA, Laing FC (1978) Anatomic variation of the portal venous anatomy in the porta hepatis: Ultrasonographic evaluation: J Clin Ultrasound, 6: 83-89.

9. Gupta SC, Gupta CD, Arora AK (1977) Intrahepatic branching patterns of portal vein. A study by corrosion casting. Gastroenterology, 72: 621-624.

10. Hu GH, Shen LG, Yang J, Mei J-H, Zhu Y-F (2008) Insight into congenital absence of the portal vein: is it rare? World J Gastroenterol, 14: 5969-5979.

11. Koc Z, Oguzkurt L, Ulusan S (2007) Portal vein variations: clinical implications and frequencies in routine abdominal multidetector CT. Turkish Soc Radio, 13: 75-81.

12. Kwok C. P, Wai FN, Christine SL, Polly PT, Asma F (2003) Anatomy of the portal vein bifurcation: Implications for transjugular Intrahepatic Portal Systemic Shunts. Cardiovasc Intervent Radiol, 26: 261-264.
13. Madoff DC, Hicks ME, Vauthey JN, Charnsaanavej C, Morello FA Jr, AhrarK, Wallace MJ, Gupta S (2002). Transhepatic portal vein embolization: anatomy, indications, and technical considerations. Radiographics, 22: 1063-1076.

14. Murphy KD, Shirodkar NP, Joiner DR, Adrian EA (1998) Portal vein bifurcation and its relevance to transjugular portosystemic shunts. J Vasc Interv Radiol, 13: 83-88.

15. Park WK, Chang JC, Bae KK, Cho JH (1996) Anatomical variation of the main and right portal vein on indirect portogram: Correlated with CT and HepaticAngiogram. J Korean Radiol Soc, 35: 231-221.

16. Schmidt S, Demartines N, Soler L, Schnyder P, Denys $A$ (2008) Portal vein normal anatomy and variants: Implication for liver surgery and portal vein embolization. Semin Intervent Radiol, 25: 86-91.

17. Schultz SR, Laberge JM, Gordon FL, Warren RS (1994) Anatomy of the portal vein bifurcation: intra-versus extrahepatic location-implications for transjugular intrahepatic portosystemic shunts. J Vasc Interv Radiol, 5: 457-459.

18. Wessa P (2008) Pearson Correlation (v1.0.3) in Free Statistics Software (v1.1.23-r7), Office for Research Development and Education, URL http://www.wessa.net/ rwasp_correlation.wasp/. 